\title{
Über die Anwendung einiger organischer Säuren zur Bestimmung von Vanadin.
}

\author{
Von \\ Phimip E. Browning und Rrchard J. Goodman. ${ }^{1}$
}

In einer früheren Arbeit ${ }^{2}$ hat der eine von uns eine Methode für die Bestimmung des Vanadins veröffentlicht, bei der Weinsäure angewendet wurde, um die Vanadinsäure zu Vanadintetroxyd zu reduzieren. Die Methode ist in kurzen Zügen die folgende:

Abgemessene Volumina einer Ammoniumvanadatlösung, deren Gehalt durch Verdampfen abgemessener Mengen und Wägen des Rückstandes bestimmt war, wurden mit Weinsäure im Überschul's gekocht, bis die auftretende tiefblaue Farbe die Reduktion der Vanadinsäure zu Vanadintetroxyd anzeigte. Nach dem Abkühlen wurde die saure Flüssigkeit schwach mit Natriumbikarbonat übersättigt, zu der alkalischen Lösung ein Überschufs einer titrierten Jodlösung hinzugefügt und dann ca. 1 Stunde stehen gelassen, bis keine weitere Entfärbung der Jodlösung eintrat. ${ }^{3}$ Das überschüssige Jod wurde mit einer titrierten Lösung von arseniger Säure zurückgenommen und schliefslich unter Stärkezusatz mit Jod auf blau zurücktitriert.

Die ganze angewendete Jodmenge, vermindert um den der arsenigen Säure entsprechenden Betrag, giebt die für die Oxydation von $\mathrm{V}_{2} \mathrm{O}_{4}$ zu $\mathrm{V}_{2} \mathrm{O}_{5}$ nötige Jodmenge, woraus nach der folgenden Gleichung das vorhandene Vanadin berechnet werden kann.

$$
\mathrm{V}_{2} \mathrm{O}_{4}+\mathrm{J}_{2}+\mathrm{H}_{2} \mathrm{O}=\mathrm{V}_{2} \mathrm{O}_{5}+2 \mathrm{HJ} \text {. }
$$

Die vorliegende Arbeit ist zum Teil eine Anwendung der beschriebenen Methode auf die Bestimmung ron Vanadin in Gegenwart von Molybdän und Wolfram.

Dor Gehalt der verwendeteı Ammoniumvanadatlösung wurle: durch Verdampfen gemessener Volumina und Glüben des Rückstandes unter Zusint\% einiger Tropfen Salpetersäure durch Wägung

1 Ins Deutsche übertragen von J. KoppEL-Berlis.

" Diese Zeitschr. 7, 158.

s Man nimmt die ganze Operation am besten in kleinen Erlenmeyerkolben, die mit paraffingetränkten Stopfen versehen sind, vor. 
bestimmt. Das Mittel einiger gut übereinstimmender Versuche wurde als der Titer der Lösung angenommen. Unsere erste Versuchsreihe umfafst Vanadinbestimmungen nach der beschriebenen Methode, dem natürlichen Ausgangspunkt für die weiteren Arbeiten. Die Resultate folgen in der Tabelle.

\begin{tabular}{r|c|c|c|c}
\hline No. & $\begin{array}{c}\text { Angewandte } \\
\text { Menge } V_{2} O_{5}\end{array}$ & $\begin{array}{c}\text { Gefundene } \\
V_{2} O_{5}\end{array}$ & Fehler & $\begin{array}{c}\text { Augewandte } \\
\text { Menge } \\
\text { Weinsăure }\end{array}$ \\
\hline 1 & $g$ & $g$ & $g$ & $g$ \\
\hline 2 & 0.1621 & 0.1618 & -0.0003 & 2 \\
3 & 0.1620 & 0.1624 & +0.0004 & 2 \\
4 & 0.1614 & 0.1622 & +0.0008 & 2 \\
5 & 0.1619 & 0.1606 & -0.0013 & 1 \\
6 & 0.1604 & 0.1597 & -0.0007 & 2 \\
7 & 0.1618 & 0.1615 & -0.0003 & 3 \\
8 & 0.1298 & 0.1305 & +0.0007 & 1 \\
9 & 0.1294 & 0.1297 & +0.0003 & 1 \\
10 & 0.1618 & 0.1618 & \pm 0.0000 & 2 \\
11 & 0.2588 & 0.2575 & -0.0013 & 3 \\
12 & 0.2722 & 0.2726 & +0.0004 & 2 \\
& 0.3273 & 0.3269 & -0.0004 & 2 \\
\hline
\end{tabular}

Im Anschluls an diese Versuche behandelten wir eine Lösung von Natriumwolframat ebenso, wie vorher die Vanadatlösung. Wir fanden, dals durch das Kochen mit Weinsäure keine Reduktion der Wolframsäure stattfand; denn bei der Rücktitration mit arseniger Säure ergab sich, dafs die verbrauchte Menge genau der zuerst zugesetzten Jodlösung äquivalent war, dafs also kein Jodverbrauch stattgefunden hatte. Demgemäls wurde eine Reihe Bestimmungen von Vanadin in Gegenwart von Wolfram gemacht, die in der folgenden Tabelle zusammengestellt sind.

\begin{tabular}{c|c|c|c|c|c}
\hline No. & $\begin{array}{c}\text { Angewandte } \\
\mathrm{V}_{2} \mathrm{O}_{5}\end{array}$ & $\begin{array}{c}\text { Gefundene } \\
\mathrm{V}_{2} \mathrm{O}_{5}\end{array}$ & Fehler & $\begin{array}{c}\text { Natrium- } \\
\text { wolframat }\end{array}$ & Weinsäure \\
\hline $\mathrm{g}$ & $\mathrm{g}$ & $\mathrm{g}$ & $\mathrm{g}$ & $\mathrm{g}$ \\
\hline 1 & 0.1618 & 0.1615 & -0.0003 & 1 & 3 \\
2 & 0.1615 & 0.1606 & -0.0009 & 1 & 3 \\
3 & 0.1618 & 0.1624 & +0.0006 & 1 & 3 \\
4 & 0.1619 & 0.1624 & +0.0005 & 1 & 3 \\
5 & 0.1627 & 0.1623 & -0.0004 & 1 & 3 \\
6 & 0.1621 & 0.1624 & +0.0003 & 1 & 4 \\
7 & 0.2587 & 0.2574 & -0.0013 & 1 & 4 \\
8 & 0.2587 & 0.2589 & -0.0002 & 1 & 4
\end{tabular}


Die erhaltenen Resultate zeigen, dafs bei der Bestimmung von Vanadin in Gegenwart von Wolfram nach der beschriebenen Methode dies letztere Element in keiner Weise einen störenden Einflufs ausübt.

Bei dem Versuche jedoch, die Bestimmung in gleicher Weise in Gegenwart von Molybdän - als Ammonmolybdat anwesend vorzunehmen, waren die Ergebnisse mit grofsen Fehlern behaftet; und einige allein mit Molybdän angestellte Versuche zeigten, dafs die Molybdänsäure beim Kochen mit Weinsäure ziemlich stark reduziert wird. Die in der folgenden Tabelle enthaltenen Zahlen lassen dies erkennen. Bei den Versuchen 3,4 und 5 wurden die Lösungen nicht mit der Weinsäure gekocht, sondern nur auf dem Wasserbade erwärmt, obne dals dadurch jedoch die Reduktion des Molybdäns merklich vermindert wurde.

\begin{tabular}{r|c|c|c|c|c}
\hline \hline No. & $\begin{array}{c}\text { Angewandte } \\
\mathrm{V}_{2} \mathrm{O}_{5}\end{array}$ & $\begin{array}{c}\text { Gefundene } \\
\mathrm{V}_{2} \mathrm{O}_{5}\end{array}$ & Fehler & $\begin{array}{c}\text { Ammonium- } \\
\text { molybdat }\end{array}$ & Weinsäure \\
\hline 1 & $\mathrm{~g}$ & $\mathrm{~g}$ & $\mathrm{~g}$ & $\mathrm{~g}$ & $\mathrm{~g}$ \\
\hline $\mathrm{y}$ & 0.1620 & 0.1790 & +0.0170 & 1 & 2 \\
3 & 0.1624 & 0.1619 & -0.0005 & 1 & 2 \\
4 & 0.1294 & 0.1416 & +0.0253 & 1 & 2 \\
5 & 0.1296 & 0.1361 & +0.0065 & 1 & 2 \\
6 & 0.1291 & 0.1312 & +0.0021 & 1 & 2 \\
7 & 0.1293 & 0.1324 & +0.0031 & 1 & 2 \\
8 & 0.1636 & 0.1760 & +0.0124 & 1 & 2 \\
9 & 0.1640 & 0.1724 & +0.0084 & 1 & 2 \\
10 & 0.1622 & 0.1624 & +0.0002 & 1 & 3 \\
11 & 0.1622 & 0.1632 & +0.0010 & 1 & 3 \\
12 & 0.1619 & 0.1879 & +0.0260 & 1 & 3 \\
13 & 0.1292 & 0.1360 & +0.0068 & 1 & 3 \\
14 & 0.1860 & 0.1917 & +0.0057 & 1 & 3 \\
15 & 0.3274 & 0.3733 & +0.0459 & 1 & 4 \\
& 0.2324 & 0.2383 & +0.0059 & 1 & 4
\end{tabular}

In der nun folgenden Versuchsreihe wurde die Einwirkung der Weinsäure auf $\mathrm{V}_{2} \mathrm{O}_{5}$ in der Kälte untersucht. Es ergab sich, dals die Reduktion auch in diesem Falle vollständig war, wenn die Weinsäure in grofsem Überschuls vorhanden und in konz. Lösung längere Zeit einwirken konnte. Die in der folgenden Tabelle aufgefülurten Bestimmungen sind zur Bestätigung dieser Angaben ausgeführt. 


\begin{tabular}{|c|c|c|c|c|c|c|}
\hline No. & $\begin{array}{c}\text { Angewandte } \\
V_{2} O_{5} \\
g \\
\end{array}$ & $\begin{array}{c}\text { Gefundene } \\
\mathrm{V}_{2} \mathrm{O}_{5} \\
g \\
\end{array}$ & $\begin{array}{c}\text { Fehler } \\
g \\
\end{array}$ & $\begin{array}{c}\text { Zeit der Ein- } \\
\text { wirkung in } \\
\text { Tagen }\end{array}$ & $\begin{array}{c}\text { Wein- } \\
\text { säure } \\
g \\
\end{array}$ & $\begin{array}{c}\text { Gesamt- } \\
\text { volum } \\
\mathrm{cem} \\
\end{array}$ \\
\hline 1 & 0.1646 & 0.1649 & +0.0003 & 1 & 4 & 25 \\
\hline 2 & 0.1640 & 0.1606 & -0.0034 & 1 & 4 & 65 \\
\hline 3 & 0.1293 & 0.1264 & -0.0029 & 2 & 3 & 55 \\
\hline 4 & 0.1633 & 0.1628 & -0.0005 & 2 & 4 & 65 \\
\hline 5 & 0.1293 & 0.1288 & -0.0005 & 3 & 2.5 & 50 \\
\hline 6 & 0.1298 & 0.1299 & +0.0001 & 3 & 2.5 & 50 \\
\hline 7 & 0.1295 & 0.1279 & -0.0016 & 3 & 3 & 55 \\
\hline 8 & 0.1617 & 0.1597 & -0.0020 & 4 & 8 & 70 \\
\hline 9 & 0.1623 & 0.1622 & -0.0001 & 4 & 3 & 80 \\
\hline
\end{tabular}

Lösungen von Natriumwolframat und Ammoniummolybdat, die 1-4 Tage mit verschiedenen Mengen von. Weinsäure standen, zeigten keine Spur von Reduktion der Metallsäuren.

Demzufolge wurde nun eine Reihe von Vanadinbestimmungen in Gegenwart von Molybdän und Wolfram ausgeführt, bei denen die Weinsäure in der Kälte 1-4 Tage ${ }^{1}$ zur Einwirkung gelangte. Es mag hierbei bemerkt werden, dal's die Resultate beim eintägigen Stehen mit $\mathbf{5} \mathbf{g}$ Weinsäure meistens vollständig zufriedensteilend sind, und dafs jedoch auch eine längere Einwirkung keine nachweisbare Reduktion der Molybdänsäure hervorruft.

\begin{tabular}{|c|c|c|c|c|c|c|c|c|}
\hline No. & $\begin{array}{c}\text { Angew. } \\
\mathrm{V}_{2} \mathrm{O}_{3} \\
g\end{array}$ & $\begin{array}{c}\text { Gefiund. } \\
\mathrm{V}_{2} \mathrm{O}_{5} \\
\mathrm{~g}\end{array}$ & $\begin{array}{c}\text { Fehler } \\
g \\
\end{array}$ & $\begin{array}{c}\text { Ammon- } \\
\text { molybdat } \\
g\end{array}$ & $\begin{array}{c}\text { Natrium- } \\
\text { wolframat } \\
\mathrm{g}\end{array}$ & 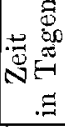 & $\begin{array}{c}\text { Gesamt- } \\
\text { volumen } \\
\text { ccm }\end{array}$ & $\begin{array}{c}\text { Wein- } \\
\text { säure } \\
\mathrm{g}\end{array}$ \\
\hline 1 & 0.1552 & 0.1558 & +0.0006 & - & - & 1 & 25 & 5 \\
\hline 2 & 0.1289 & 0.1301 & +0.0012 & - & -- & 1 & 25 & 5 \\
\hline 3 & 0.2583 & 0.2587 & +0.0004 & - & - & 1 & 50 & $\overline{5}$ \\
\hline 4 & 0.1293 & 0.1299 & +0.0006 & 1.0 & - & 1 & 25 & 6 \\
\hline 5 & 0.2582 & 0.2591 & +0.0009 & 1.0 & - & 1 & 50 & 6 \\
\hline 6 & 0.2582 & 0.2588 & +0.0006 & 1.0 & - & 1 & 50 & 5 \\
\hline 7 & 0.1297 & 0.1308 & +0.0011 & 1.0 & - & 1 & 25 & 5 \\
\hline 8 & 0.1291 & 0.1289 & -0.0002 & - & 1 & 1 & 25 & 6 \\
\hline 9 & 0.2582 & 0.2568 & -0.0014 & - & 1 & 1 & 50 & 5 \\
\hline 10 & 0.1293 & 0.1299 & +0.0006 & 1.0 & 1 & 1 & 25 & 8 \\
\hline
\end{tabular}

1 Einige dieser Versuche, bei denen die Weinsäure nach der 'Tabelle 1 Tag einwirkte, haben thatsïchlich nur 15 Stunden, von 6 Uhr Nachmittags bis 9 Uhr: Morgens, gedauert. 


\begin{tabular}{|c|c|c|c|c|c|c|c|c|}
\hline No. & $\begin{array}{c}\text { Angew. } \\
V_{2} \mathrm{O}_{5} \\
\mathrm{~g}\end{array}$ & $\begin{array}{c}\text { Gefund. } \\
\mathrm{V}_{2} \mathrm{O}_{5} \\
\mathrm{~g} \\
\end{array}$ & $\begin{array}{r}\text { Fehler } \\
8 \\
\end{array}$ & $\begin{array}{c}\text { Ammon- } \\
\text { molybdat } \\
\mathrm{g}\end{array}$ & $\begin{array}{c}\text { Natrium- } \\
\text { wolframat } \\
\mathrm{g}\end{array}$ & 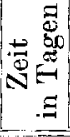 & $\begin{array}{c}\text { Gesamt- } \\
\text { volumen } \\
\text { ccm }\end{array}$ & $\begin{array}{c}\text { Wein- } \\
\text { säure } \\
\mathrm{g}\end{array}$ \\
\hline 11 & 0.2582 & 0.2579 & -0.0003 & 1.0 & 1.0 & 1 & 50 & 5 \\
\hline 12 & 0.1550 & 0.1538 & -0.0012 & - & - & 2 & 25 & 5 \\
\hline 13 & 0.1556 & 0.1545 & -0.0011 & - & - & 2 & 25 & 5 \\
\hline 14 & 0.1289 & 0.1296 & +0.0007 & - & - & 2 & 25 & 5 \\
\hline 15 & 0.1549 & 0.1527 & -0.0022 & 0.5 & - & 2 & 25 & 5 \\
\hline 16 & 0.1553 & 0.1548 & -0.0005 & 1.0 & - & 2 & 25 & 5 \\
\hline 17 & 0.1556 & 0.1554 & -0.0002 & 1.0 & - & 2 & 25 & 5 \\
\hline 18 & 0.1293 & 0.1310 & +0.0017 & 1.0 & - & 2 & 25 & 6 \\
\hline 19 & 0.1295 & 0.1299 & +0.0004 & $\cdots$ & 1.0 & 2 & 25 & 6 \\
\hline 20 & 0.1293 & 0.1289 & -0.0004 & 1.0 & 1.0 & 2 & 25 & 7 \\
\hline 21 & 0.1293 & 0.1301 & +0.0008 & - & - & 3 & 25 & 5 \\
\hline 22 & 0.1289 & 0.1299 & +0.0010 & 0.5 & - & 3 & 25 & 5 \\
\hline 23 & 0.1293 & 0.1292 & -0.0001 & 1.0 & - & 3 & 25 & 7 \\
\hline 24 & 0.1556 & 0.1567 & +0.0011 & 1.0 & - & 3 & 30 & 5 \\
\hline 25 & 0.1291 & 0.1289 & -0.0002 & 1.0 & 1.0 & 3 & 25 & 7 \\
\hline 26 & 0.1550 & 0.1557 & +0.0007 & - & - & 4 & 25 & 5 \\
\hline 27 & 0.1554 & 0.1557 & +0.0003 & 1.0 & - & 4 & 25 & 5 \\
\hline 28 & 0.1556 & 0.1557 & +0.0001 & 0.5 & - & $4:$ & 25 & 5 \\
\hline
\end{tabular}

FriedheIm und Rosenheim ${ }^{1}$ haben gezeigt, dafs Vanadinsäure durch Kochen mit Oxalsäure zu Vanadintetroxyd reduziert wird. Die Reduktion ist so vollständig, dals sie darauf eine Bestimmungsmethode für Vanadinsäure gegründet haben, die sogar in Gegenwart von Wolfram und Molybdän anwendbar ist, weil diese Metallsäuren durch Oxalsäure keine Reduktion erleiden. -

Die Oxalsäure wird hierbei oxydiert und eine bestimmte Menge Kohlensäure, gemäls der folgenden Gleichung entwickelt:

$$
\mathrm{V}_{2} \mathrm{O}_{5}+\mathrm{H}_{2} \mathrm{C}_{2} \mathrm{O}_{4}=\mathrm{V}_{2} \mathrm{O}_{4}+\mathrm{H}_{2} \mathrm{O}+2 \mathrm{CO}_{2} \text {. }
$$

Diese Kohlensäure fangen die Verfasser in geeigneten Apparaten durch Kalilauge auf und bringen sie zur Wägung. Aus ihrem Gewicht wird dann die ursprünglich vorhandene Menge der Vanadinsäure berechnet.

Wir haben diese Methode dahin abgeändert, dafs wir die nach dem Kochen mit Oxalsäure erhaltene Lösung mit einer titrierten Jodlösung genau so, wie wir es am Anfang dieser Arbeit bei der durch Weinsäure reduzierten Lösung beschrieben haben, behandelten.

1 Diese Zeitsrhr. 1, 312. 
Die in der folgenden Tabelle aufgezeichneten Resultate sind meistenteils vollkommen befriedigend und unsere Methode hat vor der von Friedheim and Rosenheim den Vorzug der gröfseren Einfachheit, da sie die Anwendung des Kaliapparates unnötig macht.

\begin{tabular}{|c|c|c|c|c|c|c|}
\hline No. & $\begin{array}{c}\underset{\mathrm{V}_{2} \mathrm{O}_{5}}{\text { Angewandite }} \\
\mathrm{g} \\
\end{array}$ & $\begin{array}{c}\text { Gefundene } \\
\mathrm{V}_{2} \mathrm{O}_{5} \\
\mathrm{~g}\end{array}$ & $\begin{array}{c}\text { Fehler } \\
\mathrm{g} \\
\end{array}$ & Oxalsäure & $\begin{array}{c}\text { Ammon- } \\
\text { molybdat } \\
\mathrm{g} \\
\end{array}$ & $\begin{array}{c}\text { Natrium- } \\
\text { wolframat } \\
\mathrm{g} \\
\end{array}$ \\
\hline 1 & 0.1806 & 0.1803 & -0.0003 & 1 & & \\
\hline 2 & 0.1950 & 0.1955 & +0.0005 & 1 & & \\
\hline 3 & 0.1959 & 0.1955 & -0.0004 & 1 & & \\
\hline 4 & 0.1950 & 0.1959 & +0.0009 & 1 & & \\
\hline 5 & 0.1954 & 0.1977 & +0.0023 & 1 & & \\
\hline 6 & 0.1956 & 0.1960 & +0.0004 & 1 & & \\
\hline 7 & 0.1956 & 0.1964 & +0.0008 & 1 & & \\
\hline 8 & 0.1956 & 0.1957 & +0.0001 & 1 & & \\
\hline 9 & 0.3900 & 0.3899 & -0.0001 & 2 & & \\
\hline 10 & 0.3897 & 0.3917 & +0.0020 & 2 & & \\
\hline 1.1 & 0.3903 & 0.3905 & +0.0002 & 2 & & \\
\hline 12 & 0.1954 & 0.1959 & +0.0005 & 2 & 1 & \\
\hline 13 & 0.1957 & 0.1960 & +0.0003 & 2 & 1 & \\
\hline 14 & 0.1954 & 0.1961 & +0.0007 & 2 & 1 & \\
\hline 15 & 0.1806 & 0.1818 & +0.0012 & 3 & - & \\
\hline 16 & 0.1807 & 0.1827 & +0.0020 & 3 & - & \\
\hline 17 & 0.1809 & 0.1803 & -0.0006 & 3 & 1 & \\
\hline 18 & 01956 & 0.1961 & +0.0005 & 3 & - & 1 \\
\hline 19 & 0.3611 & 0.3617 & +0.0006 & 5 & - & - \\
\hline 20 & 0.3616 & 0.3626 & +0.0010 & 5 & 1 & - \\
\hline
\end{tabular}

Da sich die Anwendung von Wein- und Oxalsäure für die Bestimmung von Vanadinsäure bewährt hatte, so verstand es sich von selbst, auch die. Citronensäure in derselben Weise zu benutzen, um das Studium des Verhaltens dieser Klasse von organischen Säuren gegen Vanadinsäure abzuschlieísen. Die Reduktion erfolgt auch in diesem Falle leicht und schnell; die Oxydation durch Jod dagegen geht langsamer als in Gegenwart der Alkalioxalate und -tartrate vor sich. Bei der Anwendung von Oxalsäure genügt zu ihrer Vollendung eine Viertelstunde; die Gegenwart des Tartrats erfordert etwa 30-40 Minuten zur Entfärbung der Jodlösung, während beim Citrat eine volle Stunde hierzu nötig ist.

Ein grofser Überschul's von Oxal- oder Weinsäure scheint die Resultate nicht zu beeinflussen; hingegen ist es ratsam, zu grofse Mengen von Citronensäure zu vermeiden, da hierdurch die Resultate 
leicht zu hoch ausfallen. Es muls noch bemerkt werden, dafs bei den folgenden Versuchen nie mehr als 1-2 g Citronensäure angewendet wurden, aufser in dem Falle, wo Ammoniummolybdat oder Natriumtartrat anwesend war, weil ein Teil der Basen sich mit der freien Säure verband.

\begin{tabular}{c|c|c|c|c|c|c}
\hline & $\begin{array}{c}\text { Angewendete } \\
\mathrm{V}_{2} \mathrm{O}_{5}\end{array}$ & $\begin{array}{c}\text { Gefundene } \\
\mathrm{V}_{2} \mathrm{O}_{5}\end{array}$ & Fehler & $\begin{array}{c}\text { Citronen- } \\
\text { säure }\end{array}$ & $\begin{array}{c}\text { Ammon } \\
\text { molybdat }\end{array}$ & $\begin{array}{c}\text { Natrium- } \\
\text { Wolframat }\end{array}$ \\
\hline $\mathrm{g}$ & $\mathrm{g}$ & $\mathrm{g}$ & $\mathrm{g}$ & $\mathrm{g}$ & $\mathrm{g}$ \\
\hline 1 & 0.1956 & 0.1956 & \pm 0.0000 & 1 & & \\
2 & 0.3905 & 0.3921 & +0.0016 & 2 & & \\
3 & 0.1960 & 0.1960 & \pm 0.0000 & 1 & & \\
4 & 0.1953 & 0.1960 & +0.0007 & 1 & & \\
5 & 0.2088 & 0.2082 & -0.0006 & 2 & & \\
6 & 0.2100 & 0.2098 & -0.0002 & 2 & & \\
7 & 0.2092 & 0.2107 & +0.0015 & 1 & & \\
8 & 0.2092 & 0.2107 & +0.0015 & 2 & & \\
9 & 0.2096 & 0.2082 & -0.0014 & 2 & 0.5 & \\
10 & 0.2099 & 0.2116 & +0.0017 & 3 & 0.5 & - \\
11 & 0.2005 & 0.2101 & +0.0006 & 2 & - & 0.5 \\
12 & 0.2099 & 0.2095 & -0.0004 & 3 & - & 0.5
\end{tabular}

Nach den obigen Ausführungen gestaltet sich demnach die Bestimmung der Vanadinsäure unter Anwendung von Oxal-, Weinoder Citronensäure mit kurzen Worten folgendermalsen: Zu der Lösung des Vanadats, die Molybdän und Wolfram enthalten kann, wird für jedes zehntel Gramm der zu untersuchenden Substanz annähernd $1 \mathrm{~g}$ der Säure hinzugefügt. Die Lösung wird zum Kochen erhitzt - ausgenommen, wenn bei Gegenwart von Molybdänsäure zur Reduktion Weinsäure verwendet wurde, in welchem Falle sie in der Kälte 15-20 Stunden stehen gelassen wird. $\mathrm{Zu}$ der erkalteten Flüssigkeit werden für jedes Gramm der angewendeten Säure $5 \mathrm{~g}$ Kaliumbikarbonat und sodann ein kleiner Überschufs von Jodlösung hinzugefügt. Das Gemisch bleibt stehen, bis keine weitere Entfärbung des Jods mehr wahrnehmbar ist. Das überschüssige Jod wird mit arseniger Säure zerstört, Stärke hinzugefügt und mit Jod auf blau zurücktitriert. Die Gesamtjodmenge, vermindert um das der arsenigen Säure entsprechende Äquivalent, gestattet die Berechnung des ursprünglich vorhandenen Vanadins.

Wir haben es vorteilhaft gefunden, zuerst, wenn es sich um die z. anorg. Chem. XIII. 
Bestimmung einer unbekannten Vanadatlösung handelt, eine annähernde Bestimmung zu machen, um hieraus die Mengen der für die folgenden Versuche nötigen Säure- und Jodmengen zu ermitteln. Jedoch haben wir auch bei manchen Bestimmungen einen grolsen Überschuls von Säure and von Jod verwendet, ohne einen besonders ungünstigen Einflufs auf die Resultate wahrzunehmen. Immerhin scheinen unter diesen Umständen die Werte etwas zu hoch auszufallen, was sich bei Befolgung aller angegebenen Vorschriften vollständig vermeiden lälst.

The Kent Chemical Labaratory of Yale University.

Bei der Redaktion eingegangen am 10. September 1896. 